\title{
Genetically Encoding Ultra-Stable Virus-like Particles Encapsulating Functional DNA Nanostructures in Living Bacteria
}

\section{Shai Zilberzwige-Tal}

Tel Aviv University

\section{Dan Alon}

Tel Aviv University

\section{Danielle Gazit}

Tel Aviv University

\section{Shahar Zachariah}

Tel Aviv University

\section{Amit Hollander}

Tel Aviv University

\section{Ehud Gazit}

Tel Aviv University

Johann Elbaz ( $\sim$ johannelbaz0@gmail.com )

Tel Aviv University

\section{Research Article}

Keywords: DNA Nanotechnology, Molecular-scale Device Engineering, MS2 Bacteriophage, Singlestranded DNA

Posted Date: November 9th, 2020

DOI: https://doi.org/10.21203/rs.3.rs-98920/v1

License: (c) (7) This work is licensed under a Creative Commons Attribution 4.0 International License. Read Full License 


\section{Genetically Encoding Ultra-Stable Virus-like Particles Encapsulating Functional DNA Nanostructures in Living Bacteria}

Shai Zilberzwige-Tall ${ }^{1,4}$, Dan Mark Alon ${ }^{1,4}$, Danielle Gazit ${ }^{1}$, Shahar Zachariah ${ }^{1}$, Amit Hollander ${ }^{1}$, Ehud Gazit ${ }^{1,2,3}$ and Johann Elbaz ${ }^{1^{*}}$

${ }^{1}$ The Shmunis School of Molecular Cell Biology \& Biotechnology, Faculty of Life Science, Tel Aviv University, Tel Aviv 69978, Israel.

2BLAVATNIK Center for Drug Discovery, Tel Aviv University, Tel Aviv 6997801, Israel.

${ }^{3}$ Department of Materials Science and Engineering lby and Aladar Fleischman Faculty of Engineering, Tel Aviv University, Tel Aviv 6997801, Israel.

${ }^{4}$ Equal contribution

”johanelbaz0@gmail.com

DNA nanotechnology is leading the field of in vitro molecular-scale device engineering ${ }^{1-3}$, accumulating to a dazzling array of applications from zeolite-like catalysts to bio-imaging ${ }^{1,4,5}$. However, while DNA nanostructures' function is robust under in vitro settings, their implementation in real-world conditions requires overcoming their rapid degradation and subsequent loss of function ${ }^{6,7}$. Viruses are incredibly sophisticated supramolecular assemblies, able to protect their nucleic acid content in the relatively inhospitable biological environment ${ }^{8}$. Inspired by this natural ability, we engineered both in vitro and in vivo technologies, enabling the 
encapsulation and protection of functional DNA nanostructures inside MS2 bacteriophage virus-like particles (VLPs). We demonstrate the ssDNA-VLPs nanocomposites (NCs) abilities to encapsulate single-stranded-DNA (ssDNA) of an unprecedented variety of sizes (200-1500 nucleotides (nt)), sequences, and structures while retaining their functionality ${ }^{9}$. Moreover, by exposing these NCs to hostile biological conditions, such as human blood serum, we exhibit that the VLPs serves as an excellent protective shell. To the best of our knowledge, these engineered NCs pose key properties that are yet unattainable by current fabrication methods.

\section{Main}

Based on straightforward Watson-Crick base pairing, DNA nanotechnology offers exceptional control over the shape, size, geometry, and site-specific functionalization of DNA nanostructures ${ }^{10-15}$. DNA nanostructures can be further designed as dynamic molecular devices, also known as "DNA nanomachines"1618. However, in addition to rapid degradation under real-world conditions, DNA nanotechnology suffers from certain limitations. For instance, the size of a sequence-specific ssDNA that can be chemically synthesized is limited to 200 $\mathrm{nt}^{9,19}$. Furthermore, for certain applications such as gene therapy and drug delivery, it is necessary to retain the DNA structures' functionality, a feature that is commonly overlooked when developing DNA encapsulation and delivery technologies ${ }^{20,21}$. 
Wild-type (WT) MS2 bacteriophage is a $27 \mathrm{~nm}$ particle consisting of a single copy of the maturation protein and 180 copies of the coat proteins (CP) (organized as 90 homodimers) arranged into an icosahedral shell ${ }^{22}$. The bacteriophage's assembly is driven by a specific 20 nt RNA sequence forming a stem-loop structure called "translational repression RNA" (TR-RNA), which is encoded in the MS2 genome $^{23,24}$. Recently, it has been shown that MS2 self-assembly can be initiated in vitro in the presence of a $20 \mathrm{nt}$ TR-DNA sequence analogous to the MS2 TR$\mathrm{RNA}^{25}$.

Throughout the past decade, researchers have utilized the unprecedented selfassembly properties of viruses to engineer materials at the nanometer scale for various applications such as solar cells ${ }^{26}$, batteries ${ }^{27}$, medical diagnostics ${ }^{28}$, regenerative medicine ${ }^{29}$, gene therapy ${ }^{30}$, and drug delivery platforms ${ }^{31}$. However, utilizing viruses for shielding functional DNA structures under complex conditions has not been demonstrated yet. We report both in vitro and in vivo technologies for the production of functional ssDNA-VLPs NCs that meet the requirements of ssDNA protection and functionality under real-world conditions, whereas the MS2 VLPs are the shield and the functional DNA is the cargo. The in vitro technology can produce MS2 VLPs encapsulating chemically synthesized ssDNAs in the form of a DNA light-up aptamer and a DNA nanotweezers (Figure 1a). In the in vivo technology, we genetically encoded the integration of ssDNA production with the expression of MS2 CP in vivo, resulting in the self-assembly of novel functional ssDNA-VLPs NCs within living bacteria (Figure 1b). 
First, we aimed to encapsulate a DNA nanostructure in the form of a "light-up" aptamer (see methods) which can be used as a sensor. MS2 CP dimers were purified from $E$. coli and incubated in the presence of the ssDNA ${ }^{32,33}$. The ssDNA included the TR-DNA and Malachite Green "light-up" DNA aptamer ${ }^{33}$ (MGA) sequences (Figure 2a, Figure S1). Following incubation, ssDNA-VLPs NCs were characterized by using transmission electron microscopy (TEM), showing that the sSDNA-VLPs NCs were of $\sim 30 \mathrm{~nm}$ in diameter (Figure $2 \mathrm{~b}$, Figure S3-S6), similar to WT MS2. To test the hypothesis that the MGA remains active inside the ssDNAVLPs NCs, Malachite Green dye was added to the samples and high fluorescence signal was measured (Figure 2c). Thus, the MGA ssDNA was able to fold into an active secondary structure and specifically bind Malachite Green dye molecules, while encapsulated within the MS2 VLPs, forming novel active NCs. As expected, the encapsulated MGA fluorescence signal preservation ratio post-DNase treatment was higher than the unencapsulated MGA (Figure 2c). While the functional MGA encapsulated within the MS2 VLPs maintained $61 \%$ of the initial fluorescence signal following DNase treatment, the fluorescence of the unencapsulated MGA dramatically decreased to $20 \%$. This emphatically indicates that, similar to bacteriophages, the MS2 ssDNA-VLPs NCs can both encapsulate and protect nucleic acid cargo and that the NCs can maintain ssDNA activity. The success of producing active ssDNA-VLPs NCs has motivated us to produce more intricate NCs: MS2 VLPs encapsulating functional pH-dependent DNA nanotweezers ${ }^{34}$. The nanotweezers are responsive to a specific environmental 
trigger (see methods) and it was previously reported that WT MS2 maintains its stability under these conditions ${ }^{35}$. We, therefore, designed three different variants of the DNA nanotweezers where each variant includes the TR-DNA activator sequence at a different location (Figure S2). The DNA nanotweezers contained a fluorophore (Cy5) at the 5' end and a fluorescence quencher (AbRQSp) at the 3' end. Thus, under neutral pH, the DNA nanotweezers is in an "open" state and there is a high fluorescence signal. However, under acidic conditions, the tweezer undergoes a conformational change to a closed state, positioning the fluorophore and the fluorescence quencher in close proximity and thus reducing the fluorescence signal (Figure 3a). First, the nanotweezers were assembled in vitro and MS2 CPs were then added, resulting in the self-assembly of the ssDNA-VLPs NCs. The NCs were analyzed using TEM, where the NCs were observed and found to be $\sim 30 \mathrm{~nm}$ in diameter, similar to WT MS2 (Figure 3d, Figure S7-S10). Hence, complex DNA nanomachines such as DNA nanotweezers can be encapsulated inside MS2 VLPs, resulting in novel tweezers-VLPs NCs.

Next, the tweezers-VLPs NCs functionality was confirmed. This was done by measuring the fluorescence signal under both neutral and acidic conditions $(\mathrm{pH}$ 7.3 and $\mathrm{pH} 5.3$, respectively, see methods) following a DNase I treatment. Under neutral $\mathrm{pH}$ conditions, the tweezers-VLPs NCs produced a significant fluorescence signal, whereas a low fluorescence signal was observed in the control group (unencapsulated DNA nanotweezers) (Figure 3b and c, Figures S11-S13). These results suggest that the VLPs shell was able to both maintain the nanotweezers structure-activity and to protect it from degradation. Under acidic conditions, both 
the tweezers-VLPs NCs and the control group showed reduced fluorescence signal. Hence, the nanotweezers maintained autonomous functionality inside the VLPs and the tweezers-VLPs NCs acted as a pH sensor.

Our next goal was to mass-produce ssDNA-VLPs NCs in vivo. The capability to synthesize any ssDNA sequence and encapsulate it inside a protective shell, such as the MS2 VLPs, holds great potential in nanotechnology for various applications. However, a synthetic genetic system dictating the encapsulation of any ssDNA within VLPs in living cells has not yet been shown. We recently engineered a system that utilizes the human immunodeficiency virus reverse transcriptase (HIVRT) and murine leukemia reverse transcriptase (MLRT) to produce ssDNA in vivo $^{36,37}$. This technology can specifically convert engineered non-coding RNAs (r_oligo) into a ssDNA of choice. We hypothesize that this ssDNA production technology can be re-engineered to initiate ssDNA-VLPs NCs assembly in vivo (Figure 4a). The TR-DNA activator sequence was added to the 3' of the produced ssDNA and co-expressed with MS2 CP dimers in E. coli. It should be noted that the addition of the TR-DNA activator sequence to the 3 ' end of the SsDNA produced in vivo, eliminates the potential encapsulation of non-desired SSDNA intermediates (Figure 4a).

We aimed to surpass the 200-nt ssDNA production limit and the engineered r_oligo was designed at different lengths ranging from 200 to 1500 -nt. The purified samples were analyzed using TEM and Cryo-TEM, where MS2 VLPs of $\sim 30 \mathrm{~nm}$ diameter (similar to WT MS2) were observed only in the presence of all the 
system's parts (RTs, CPs, r_oligo) (Figure 4b). The deletion of the TR-DNA sequence from the r_oligo, the absence of the CP, or the absences of the ssDNA prevented the ssDNA-VLPs NCs' assembly (Figure S14). To evaluate the length of the encapsulated ssDNA, the ssDNA was purified directly from the ssDNA-VLPs NCs and visualized using gel electrophoresis (Figure 4c). This result suggests that the ssDNA-VLPs NCs produced in vivo can produce sSDNA, which significantly exceeds the 200-nt limit. Furthermore, the ssDNA production yield from the ssDNA-VLPs NCs was evaluated to be $27 \mathrm{mg}$ ssDNA/L of bacteria and $2.5^{\star} 10^{\wedge} 11$ VLPs/mL (see methods). These yields are more than 1000-fold higher than the purification yields of unencapsulated ssDNA for $1 \mathrm{~L}$ of bacteria ${ }^{36}$.

The purified ssDNA was further characterized by Sanger sequencing; as expected, sequencing results correspond to the engineered desired ssDNA sequence (Figure 4d). The ssDNA encapsulated inside the MS2 VLPs is not limited in its sequence as long as the TR-DNA activator sequence remains in the 3' end. Linear DNA is highly prone to degradation under cellular conditions and, therefore, needs to be designed in structures or to be chemically modified in order to reduce its degradation rate. However, with our technology the VLPS' shell protects the DNA under cellular conditions and in the presence of DNase (see methods). As a result, in the described in vivo technology, the ssDNA does not require additional structural design or chemical modifications to increase its stability in vivo. Thus, the newly developed technology was able to genetically encode, produce and encapsulate long ssDNA inside MS2 VLPs in vivo. 
We examined the ability of the in vivo technology to encapsulate functional DNA structures inside the MS2 VLPs. For this purpose, as a reporter element, we used the MGA "light-up" aptamer, which was successfully used in the in vitro technology. We have incorporated the MGA sequence into the r_oligo gene together with the TR-DNA activator sequence (Figure 5). By measuring the MGA fluorescence signal we were able to determine whether the encapsulated ssDNA maintained its structural folding properties. Aptamer-VLPs NCs were purified from cells; then, Malachite Green dye was added to the samples. As expected, a strong fluorescence signal was observed in the aptamer-VLPs NCs, whereas in the sample containing VLPs encapsulating random ssDNA sequences no fluorescence signal was observed (Figure 5a). This confirms that the precise DNA structure can fold within the aptamer-VLPs NCs and further interact with the surrounding environment as Malachite Green dye molecules can diffuse into the VLPs.

Next, we tested the possibility that the encapsulation of the structural DNA inside the VLPs will increase the fluorescence signal in vivo as the VLPs' shell can protect the encapsulated ssDNA from the nuclease-rich cellular environment. The in vivo technology was expressed in E. coli; following expression, Malachite Green dye was added to the culture and fluorescence measurements were conducted (Figure 5a). A strong fluorescence signal was observed from the bacteria expressing the ssDNA-VLPs NCs with the MGA sequence, while bacteria expressing the ssDNAVLPs NCs with random ssDNA sequences, produced a significantly lower signal. 
This result indicates that the MGA can fold within the VLPs NC in vivo and maintain its functionality in comparison to random ssDNA encapsulation. Thus, the MGA can indeed act as a reporter element of the in vivo technology. When compared to free aptamer within bacteria (lacking the expression of the MS2 CP dimers), the aptamer-VLPs NCs exhibit a strong fluorescence signal (Figure 4b). Thus, aptamer-VLPs NCs produce a protective shell against the hostile cellular environment of $E$. coli and preserve the aptamer's structure and function in vivo. The protection of DNA structures under cellular conditions may lead to the engineering and production of various DNA sensors and nanomachines in vivo for medical and on-soil sensing.

As a means to study the importance of the TR-DNA sequence for the VLPs selfassembly process, we designed four different r_oligo with 2-5-nt deletions in their TR-DNA sequence (Figure S17). Fluorescence measurements were performed on living bacteria expressing the different variants. The high fluorescence signal was detected in the WT TR-DNA variant and a decrease in the fluorescence signal was observed in correlation with the number of nucleotides that were deleted from the TR-DNA sequence (Figure 5b). We suggest that the deletion of these nucleotides may prevent the formation of the TR-DNA bulge that contains a free adenine nucleotide, which was previously reported to be a key structural feature in the TRRNA recognition and the self-assembly process of WT MS2 bacteriophages ${ }^{23,24}$. 
A major goal of DNA nanotechnology is to engineer molecular tools that can operate in harsh biological environments, enabling drug delivery or diagnostic functionality. However, it is well known that free ssDNA is highly prone to degradation under various biological environments and its functionality is severely limited in cell culture medium with $10 \%$ fetal bovine serum (FBS) ${ }^{6}$. Moreover, exogenously delivered nucleic acids can trigger an innate immune response through the activation of toll-like receptors (TLRs) ${ }^{6,38}$. The novel ssDNA-VLPs NCs can serve as a new nanotechnology tool that can be applied under these natural environments and real-world conditions. As a proof of principle, free aptamer and aptamer-VLPs NCs were incubated with $100 \%$ human blood serum, which is a nuclease rich environment ${ }^{7}$. Aliquots from the samples were collected at different time points and fluorescence measurements were conducted (Figure $5 c$ ). These results suggest that aptamer-VLPs NCs were able to maintain the fluorescence signal with more than $60 \%$ preservation ratio following $1 \mathrm{hr}$ incubation with $100 \%$ human blood serum. In contrast, the free aptamer fluorescence signal rapidly decreased with less than $50 \%$ preservation ratio following 20 minutes incubation. Therefore, the aptamer-VLPs NCs were significantly more stable than the free MGA, showing a protective quality of the VLPs under real-world conditions. These results demonstrate the potential of this technology to mass-produce NCs that can serve both as functional particles under harsh biological environments and as delivery platforms of operational DNA nanostructures.

\section{Conclusion}


Inspired by the bacteriophage's reproduction mechanism and its ability to protect its nucleic acid content in endonucleases rich environments, we developed new technologies for the production of novel and functional ssDNA-VLPs NCs. The ssDNA-VLPs NCs present diverse ssDNA sizes, sequences, structures, and functionalities. Moreover, the size of ssDNA sequences that can be produced using this technology is greater than what can be chemically synthesized today (more than 7-fold). The high DNA purification yields hold great potential for the development of a cheap and smart infrastructure for the production of DNA and RNA nanotechnology. Furthermore, this technology offers the ability to generate highly durable NCs encapsulating functional DNA nanomachines, which can also serve as a targeted drug delivery platform $30,31,39,40$.

In conclusion, the reported technologies can serve as one of the missing links between in vitro experiments and real-world applications of DNA nanotechnology.

\section{Acknowledgments}

We thank Dafna Greitzer for her help with graphical design. We also thank Dr. Naama Koifman from The Technion Center for Electron Microscopy of Soft Matter for preforming the Cryo-TEM images. In addition, we thank the Gazit and Elbaz lab members for fruitful discussions.

\section{Author Contributions}

S.Z-T. performed the experiments. D.A, D.G., S.Z, and A. H participated in part of the experiments. D. A designed and analyzed some of the experiments and edited 
the manuscript. S. Z.-T., and J.E. conceived the project, analyzed data, and wrote the manuscript. E.G. conceived the use of the MS2 technology, analyzed and edited the manuscript.

\section{Funding sources}

This study was supported by the ISF Foundation, grant number 0604516882, and by the Adelis Foundation.

\section{References}

1 Seeman, N. C. \& Sleiman, H. F. DNA nanotechnology. Nature Reviews Materials 3, doi:10.1038/natrevmats.2017.68 (2017).

2 Xavier, P. L. \& Chandrasekaran, A. R. DNA-based construction at the nanoscale: emerging trends and applications. Nanotechnology 29, 062001, doi:10.1088/1361-6528/aaa120 (2018).

$3 \quad$ Ke, Y., Ong, L. L., Shih, W. M. \& Yin, P. Three-dimensional structures selfassembled from DNA bricks. Science 338, 1177-1183, doi:10.1126/science.1227268 (2012).

4 Meng, H. M. et al. Aptamer-integrated DNA nanostructures for biosensing, bioimaging and cancer therapy. Chem Soc Rev 45, 2583-2602, doi:10.1039/c5cs00645g (2016).

5 Madsen, M. \& Gothelf, K. V. Chemistries for DNA Nanotechnology. Chem Rev 119, 6384-6458, doi:10.1021/acs.chemrev.8b00570 (2019). 
6 Chen, Y. J., Groves, B., Muscat, R. A. \& Seelig, G. DNA nanotechnology from the test tube to the cell. Nat Nanotechnol 10, 748-760, doi:10.1038/nnano.2015.195 (2015).

7 Conway, J. W., McLaughlin, C. K., Castor, K. J. \& Sleiman, H. DNA nanostructure serum stability: greater than the sum of its parts. Chem Commun (Camb) 49, 1172-1174, doi:10.1039/c2cc37556g (2013).

8 Forterre, P. \& Prangishvili, D. The great billion-year war between ribosomeand capsid-encoding organisms (cells and viruses) as the major source of evolutionary novelties. Ann N Y Acad Sci 1178, 65-77, doi:10.1111/j.1749$6632.2009 .04993 . x(2009)$.

9 Kosuri, S. et al. Scalable gene synthesis by selective amplification of DNA pools from high-fidelity microchips. Nat Biotechnol 28, 1295-1299, doi:10.1038/nbt.1716 (2010).

10 Winfree, E., Liu, F., Wenzler, L. A. \& Seeman, N. C. Design and selfassembly of two-dimensional DNA crystals. Nature 394, 539-544, doi:10.1038/28998 (1998).

11 Seeman, N. C. Nucleic acid junctions and lattices. J Theor Biol 99, 237-247 (1982).

12 Wang, Z. G. \& Ding, B. Engineering DNA self-assemblies as templates for functional nanostructures. Acc Chem Res 47, 1654-1662, doi:10.1021/ar400305g (2014).

13 Kocar, V. et al. Design principles for rapid folding of knotted DNA nanostructures. Nat Commun 7, 10803, doi:10.1038/ncomms10803 (2016). 
14 Thum, O., Jager, S. \& Famulok, M. Functionalized DNA: A New Replicable Biopolymer. Angew Chem Int Ed Engl 40, 3990-3993, doi:10.1002/15213773(20011105)40:21<3990::AID-ANIE3990>3.0.CO;2-O (2001).

15 Sun, W. et al. Precise pitch-scaling of carbon nanotube arrays within threedimensional DNA nanotrenches. Science 368, 874-877, doi:10.1126/science.aaz7440 (2020).

16 Wang, Z. G., Elbaz, J. \& Willner, I. DNA machines: bipedal walker and stepper. Nano Lett 11, 304-309, doi:10.1021/nl104088s (2011).

17 Yin, P., Yan, H., Daniell, X. G., Turberfield, A. J. \& Reif, J. H. A Unidirectional DNA Walker That Moves Autonomously along a Track. Angewandte Chemie 116, 5014-5019, doi:10.1002/ange.200460522 (2004).

18 Lohmann, F., Ackermann, D. \& Famulok, M. Reversible light switch for macrocycle mobility in a DNA rotaxane. J Am Chem Soc 134, 11884-11887, doi:10.1021/ja3042096 (2012).

19 Hao, M., Qiao, J. \& Qi, H. Current and Emerging Methods for the Synthesis of Single-Stranded DNA. Genes (Basel) 11, doi:10.3390/genes11020116 (2020).

20 Meng, L. et al. Tetrahedral DNA Nanostructure-Delivered DNAzyme for Gene Silencing to Suppress Cell Growth. ACS Appl Mater Interfaces 11, 6850-6857, doi:10.1021/acsami.8b22444 (2019).

21 Zhang, Q. et al. DNA origami as an in vivo drug delivery vehicle for cancer therapy. ACS Nano 8, 6633-6643, doi:10.1021/nn502058j (2014). 
22 Koning, R. I. et al. Asymmetric cryo-EM reconstruction of phage MS2 reveals genome structure in situ. Nat Commun 7, 12524, doi:10.1038/ncomms12524 (2016).

23 Peabody, D. S. The RNA binding site of bacteriophage MS2 coat protein. The EMBO journal 12, 595-600 (1993).

24 Horn, W. T. et al. The crystal structure of a high affinity RNA stem-loop complexed with the bacteriophage MS2 capsid: further challenges in the modeling of ligand-RNA interactions. Rna 10, 1776-1782, doi:10.1261/rna.7710304 (2004).

25 Glasgow, J. E., Capehart, S. L., Francis, M. B. \& Tullman-Ercek, D. Osmolyte-mediated encapsulation of proteins inside MS2 viral capsids. ACS Nano 6, 8658-8664, doi:10.1021/nn302183h (2012).

26 Dang, X. et al. Virus-templated self-assembled single-walled carbon nanotubes for highly efficient electron collection in photovoltaic devices. Nat Nanotechno/ 6, 377-384, doi:10.1038/nnano.2011.50 (2011).

27 Nam, K. T. et al. Virus-enabled synthesis and assembly of nanowires for lithium ion battery electrodes. Science 312, 885-888, doi:10.1126/science.1122716 (2006).

28 Schwarz, B. \& Douglas, T. Development of virus-like particles for diagnostic and prophylactic biomedical applications. Wiley Interdiscip Rev Nanomed Nanobiotechnol 7, 722-735, doi:10.1002/wnan.1336 (2015).

29 Pang, H. H. et al. Convection-Enhanced Delivery of a Virus-Like Nanotherapeutic Agent with Dual-Modal Imaging for Besiegement and 
Eradication of Brain Tumors. Theranostics 9, 1752-1763, doi:10.7150/thno.30977 (2019).

30 Wu, M., Sherwin, T., Brown, W. L. \& Stockley, P. G. Delivery of antisense oligonucleotides to leukemia cells by RNA bacteriophage capsids. Nanomedicine 1, 67-76, doi:10.1016/j.nano.2004.11.011 (2005).

31 Galaway, F. A. \& Stockley, P. G. MS2 viruslike particles: a robust, semisynthetic targeted drug delivery platform. Mol Pharm 10, 59-68, doi:10.1021/mp3003368 (2013).

32 Zilberzwige-Tal, S., Levin, A., Toprakcioglu, Z., Knowles, T., Gazit, E. \& Elbaz, J. Programmable On-Chip Artificial Cell Producing PostTranslationally Modified Ubiquitinated Protein. Small 15, 1901780 (2019).

33 Wang, $\mathrm{H}$. et al. Selection and Characterization of Malachite Green Aptamers for the Development of Light-up Probes. ChemistrySelect 1, 1571-1574, doi:10.1002/slct.201600154 (2016).

34 Modi, S. et al. A DNA nanomachine that maps spatial and temporal $\mathrm{pH}$ changes inside living cells. Nat Nanotechnol 4, 325-330, doi:10.1038/nnano.2009.83 (2009).

35 Feng, Y. Y., Ong, S. L., Hu, J. Y., Tan, X. L. \& Ng, W. J. Effects of pH and temperature on the survival of coliphages MS2 and Qbeta. J Ind Microbiol Biotechno/ 30, 549-552, doi:10.1007/s10295-003-0080-y (2003).

36 Alon, D. M., Voigt, C. A. \& Elbaz, J. Engineering a DNAzyme-Based Operon System for the Production of DNA Nanoscaffolds in Living Bacteria. ACS Synth Biol 9, 236-240, doi:10.1021/acssynbio.9b00415 (2020). 
37 Elbaz, J., Yin, P. \& Voigt, C. A. Genetic encoding of DNA nanostructures and their self-assembly in living bacteria. Nat Commun 7, 11179, doi:10.1038/ncomms11179 (2016).

38 Panter, G., Kuznik, A. \& Jerala, R. Therapeutic applications of nucleic acids as ligands for Toll-like receptors. Curr Opin Mol Ther 11, 133-145 (2009).

39 Wu, M., Brown, W. L. \& Stockley, P. G. Cell-specific delivery of bacteriophage-encapsidated ricin A chain. Bioconjug Chem 6, 587-595 (1995).

40 Ashley, C. E. et al. Cell-specific delivery of diverse cargos by bacteriophage MS2 virus-like particles. ACS Nano 5, 5729-5745, doi:10.1021/nn201397z (2011).

Fig. 1: The design of both the in vitro and the in vivo systems for the production of ssDNA-VLPs NCs. (a) The in vitro production mechanism is shown. The CP dimers (blue) are purified from E.coli and then incubated with the DNA nanomachine conjugated to the TR-DNA activator sequence (red). (b) The in vivo production mechanism is shown. The system consists of: (i) ssDNA production from the oligo gene (see Fig. 4a for more details), and (ii) the expression of the CP dimers (blue). The oligo gene is designed to include at its 3 ' end the TR- 
DNA activator sequence (red) leading to the VLPs' self-assembly and the encapsulation of the ssDNA within the VLPs. Following their self-assembly, the ssDNA-VLPs NCs are purified from the expressing bacteria.

Fig. 2: In vitro production of functional ssDNA-VLPs NCs. (a) Schematic representation of the protective quality of the ssDNA-VLPs NCs against nucleases. Blue - MS2 CP dimers are incubated with DNA "light-up" aptamer conjugated to the TR-DNA activator sequence (red), resulting in the self-assembly of the ssDNAVLPs NCs. The DNA "light-up" aptamer remains active inside the NC and specifically binds the Malachite green molecule. Following exposure to DNase I, the DNA "light-up" aptamer remains active inside the VLPs. Grey unencapsulated DNA "light-up" aptamer binds Malachite Green molecule. Following exposure to DNase I, the Malachite Green DNA aptamer is degraded and cannot bind the Malachite Green molecule. (b) TEM image of the in vitro selfassembled aptamer-VLPs NCs. (c) The fluorescence preservation ratio of the unencapsulated and encapsulated DNA aptamer post-exposure to DNase I is shown.

Fig. 3: In vitro assembly of ultra-stable functional NCs comprising of MS2 VLPs encapsulating DNA nanotweezers. (a) Schematic representation of the tweezers-VLPs NCs; The three oligos (A1-A3) comprising the DNA tweezers are designed as follows: A1 is conjugated to a fluorophore (Cy5) (designated as F) at its 5 ' end. A2 is conjugated to a quencher (AbRQSp) (designated as Q) at its 3' 
end. A3 includes within its sequence the TR-DNA activator sequence at its hinge point (red). Following the in vitro assembly of the DNA nanotweezers, MS2 CP dimers are added, resulting in the encapsulation of the DNA nanotweezers within the MS2 VLPs. At $\mathrm{pH}=7$, the DNA nanotweezers is in an open state, which is indicated by a strong fluorescence signal. Under acidic conditions $(\mathrm{pH} 5)$, the DNA nanotweezers is closed which is indicated by a decrease in the fluorescence signal. (b) Fluorescence spectra of the tweezers-VLPs NCs at $\mathrm{pH}=7.3$ (green), and $\mathrm{pH}=5.3$ (orange), in an open and closed state respectively. (c) The fluorescence signal of the encapsulated and unencapsulated DNA nanotweezers post-DNase incubation. ${ }^{* *} \mathrm{P}<0.005$ (2-tailed Student's t-test). Error bars represent full experimental reproduction (3 repeats) of the process. (d) TEM image of the tweezers-VLPs NCs.

Fig. 4. In vivo production of ssDNA-VLPs NCs. (a) Schematic representation of the genetically encoded in vivo production system's mechanism. The r_oligo gene contains (i) the desired ssDNA sequence, such as an aptamer (black), (ii) the TRDNA activator sequence (red), (iii) the HIV reverse transcriptase binding site (HTBS), which serves as a terminator (grey), and (iv) self-cleaved DNAzyme which removes the HTBS following the conversion from RNA to DNA. (b) TEM images of the in vivo produced ssDNA-VLPs NCs. The inset represents the Cryo-TEM image of the same sample. (c) Gel electrophoresis of ssDNA sequences of different lengths, which were purified directly from the ssDNA-VLPs NCs. (d) 
Representative sequencing analysis of the in vivo produced ssDNA. The redmarked sequence represents the TR-DNA sequence.

Fig. 5. Application of the ssDNA-VLPs NCs under real-world conditions. (a) The fluorescence signal of functional aptamer-VLPs NCs within bacteria (left), and post purification (right). (b) Fluorescence signals of the TR-DNA variants showing the impact of the TR-DNA activator sequence on ssDNA-VLPs NCs' assembly. ${ }^{*} \mathrm{P}<0.01, \quad{ }^{*} \mathrm{P}<0.005, \quad$ (2-tailed Student's t-test). Error bars represent full experimental reproduction (3 repeats) of the process. (c) Comparison between the fluorescence signals at different time points of encapsulated MGA inside ssDNA-VLPs NCs and free MGA in $100 \%$ human blood serum. 


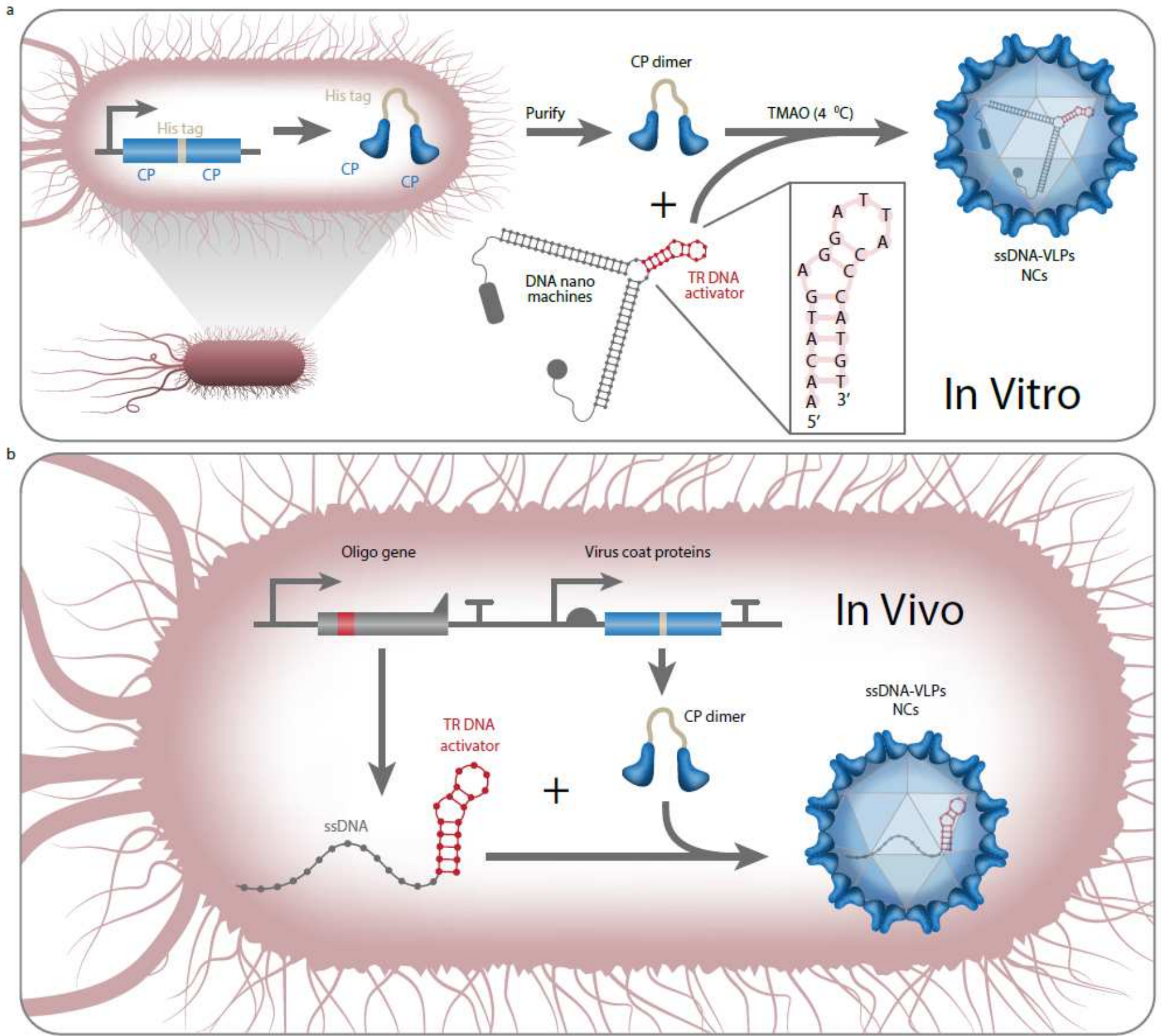

\section{Figure 1}

The design of both the in vitro and the in vivo systems for the production of sSDNA-VLPs NCs. (a) The in vitro production mechanism is shown. The CP dimers (blue) are purified from E.coli and then incubated with the DNA nanomachine conjugated to the TR-DNA activator sequence (red). (b) The in vivo production mechanism is shown. The system consists of: (i) ssDNA production from the oligo gene (see Fig. 4a for more details), and (ii) the expression of the CP dimers (blue). The oligo gene is designed to include at its $3^{\prime}$ end the TR-DNA activator sequence (red) leading to the VLPs' self-assembly and the 
encapsulation of the ssDNA within the VLPs. Following their self-assembly, the ssDNA-VLPs NCs are purified from the expressing bacteria.

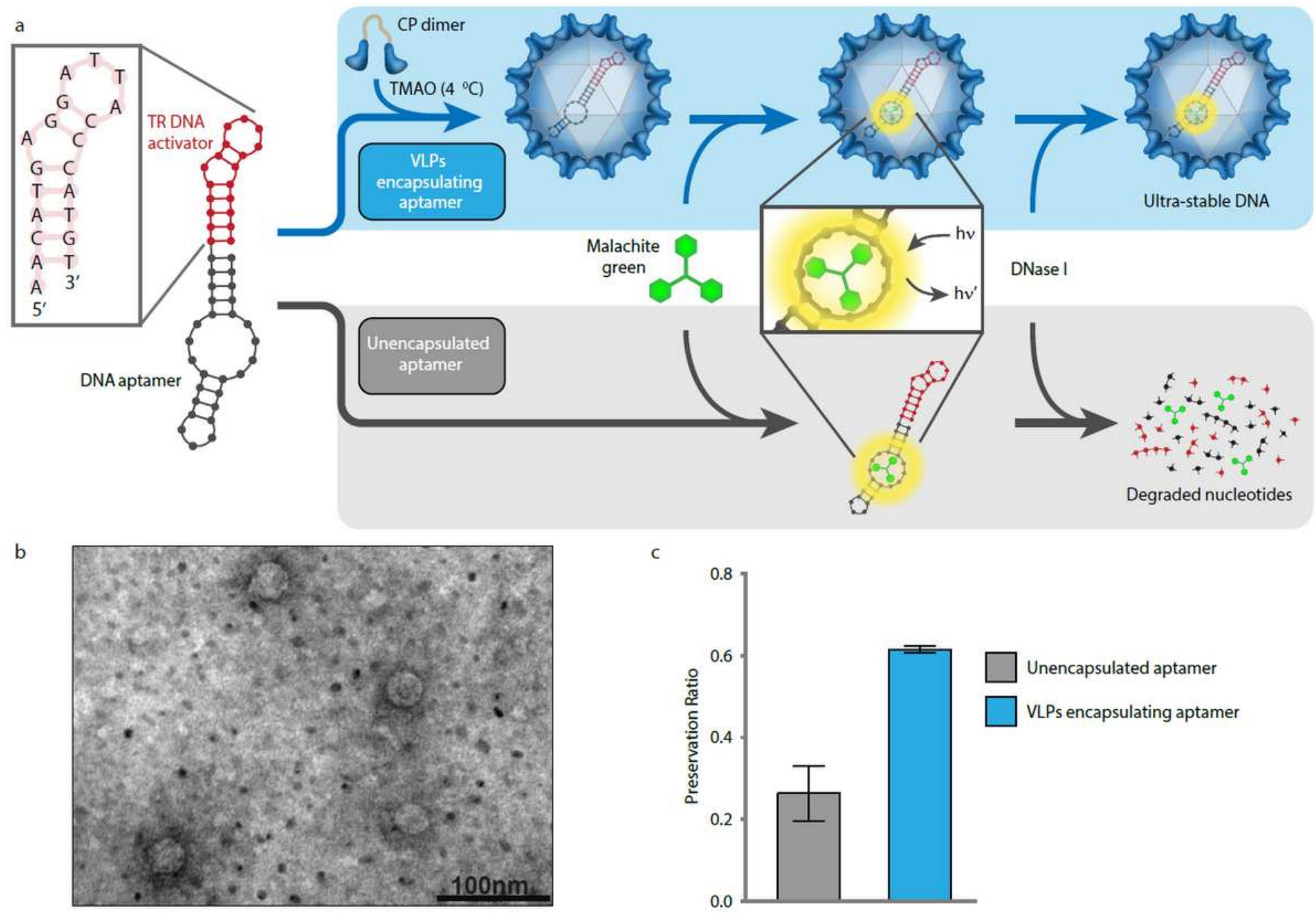

Figure 2

In vitro production of functional SSDNA-VLPs NCs. (a) Schematic representation of the protective quality of the ssDNA-VLPs NCs against nucleases. Blue - MS2 CP dimers are incubated with DNA "light-up" aptamer conjugated to the TR-DNA activator sequence (red), resulting in the self-assembly of the ssDNAVLPs NCs. The DNA "light-up" aptamer remains active inside the NC and specifically binds the Malachite green molecule. Following exposure to DNase I, the DNA "light-up" aptamer remains active inside the VLPs. Grey - unencapsulated DNA “light-up” aptamer binds Malachite Green molecule. Following exposure to DNase I, the Malachite Green DNA aptamer is degraded and cannot bind the Malachite Green molecule. (b) TEM image of the in vitro self-assembled aptamer-VLPs NCs. (c) The fluorescence preservation ratio of the unencapsulated and encapsulated DNA aptamer post-exposure to DNase I is shown. 

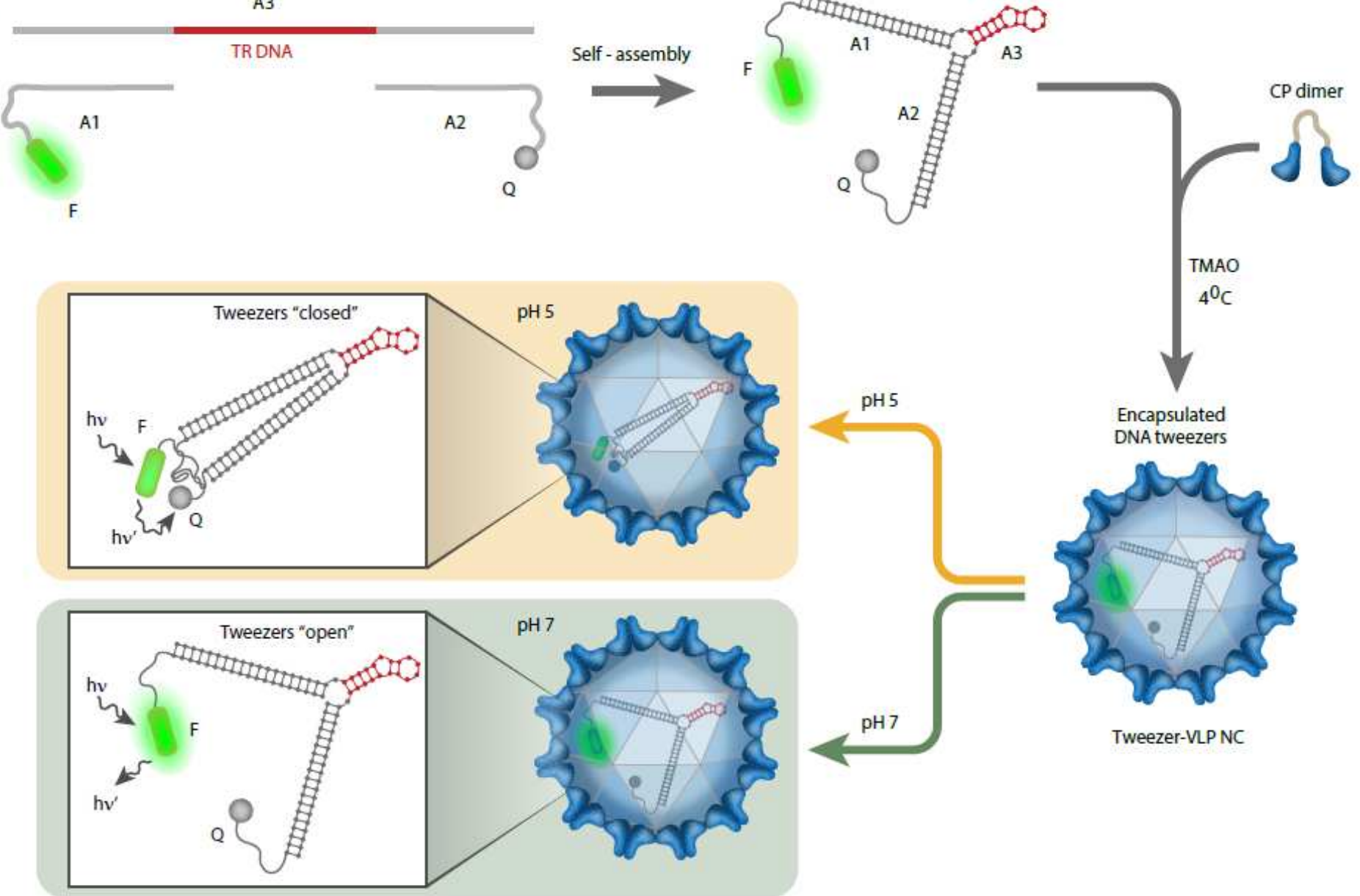

$\mathrm{pH} 7$

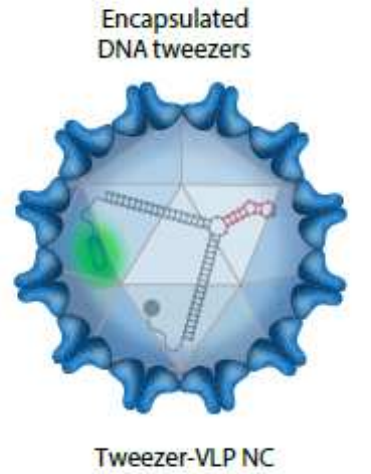

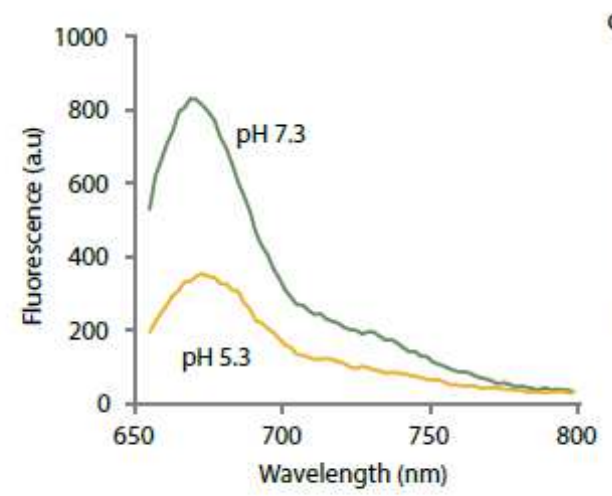

C

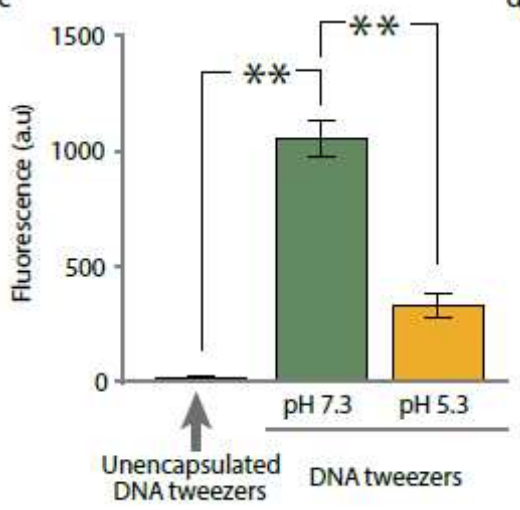

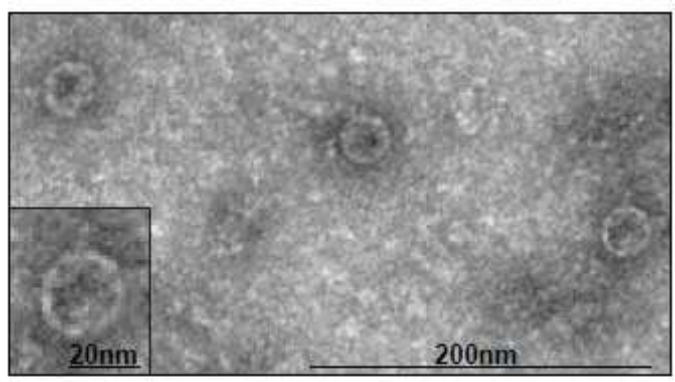

\section{Figure 3}

In vitro assembly of ultra-stable functional NCs comprising of MS2 VLPs encapsulating DNA nanotweezers. (a) Schematic representation of the tweezers-VLPs NCs; The three oligos (A1-A3) comprising the DNA tweezers are designed as follows: A1 is conjugated to a fluorophore (Cy5) (designated as F) at its $5^{\prime}$ end. A2 is conjugated to a quencher (AbRQSp) (designated as Q) at its 3' end. A3 includes within its sequence the TR-DNA activator sequence at its hinge point (red). Following the in vitro assembly of the DNA nanotweezers, MS2 CP dimers are added, resulting in the encapsulation of the DNA nanotweezers within the MS2 VLPs. At $\mathrm{pH}=7$, the DNA nanotweezers is in an open state, which is indicated by a strong fluorescence signal. Under acidic conditions ( $\mathrm{pH} 5)$, the DNA nanotweezers is closed 
which is indicated by a decrease in the fluorescence signal. (b) Fluorescence spectra of the tweezersVLPs NCs at $\mathrm{pH}=7.3$ (green), and $\mathrm{pH}=5.3$ (orange), in an open and closed state respectively. (c) The fluorescence signal of the encapsulated and unencapsulated DNA nanotweezers post-DNase incubation. $\star \star P<0.005$ (2-tailed Student's t-test). Error bars represent full experimental reproduction (3 repeats) of the process. (d) TEM image of the tweezers-VLPs NCs.

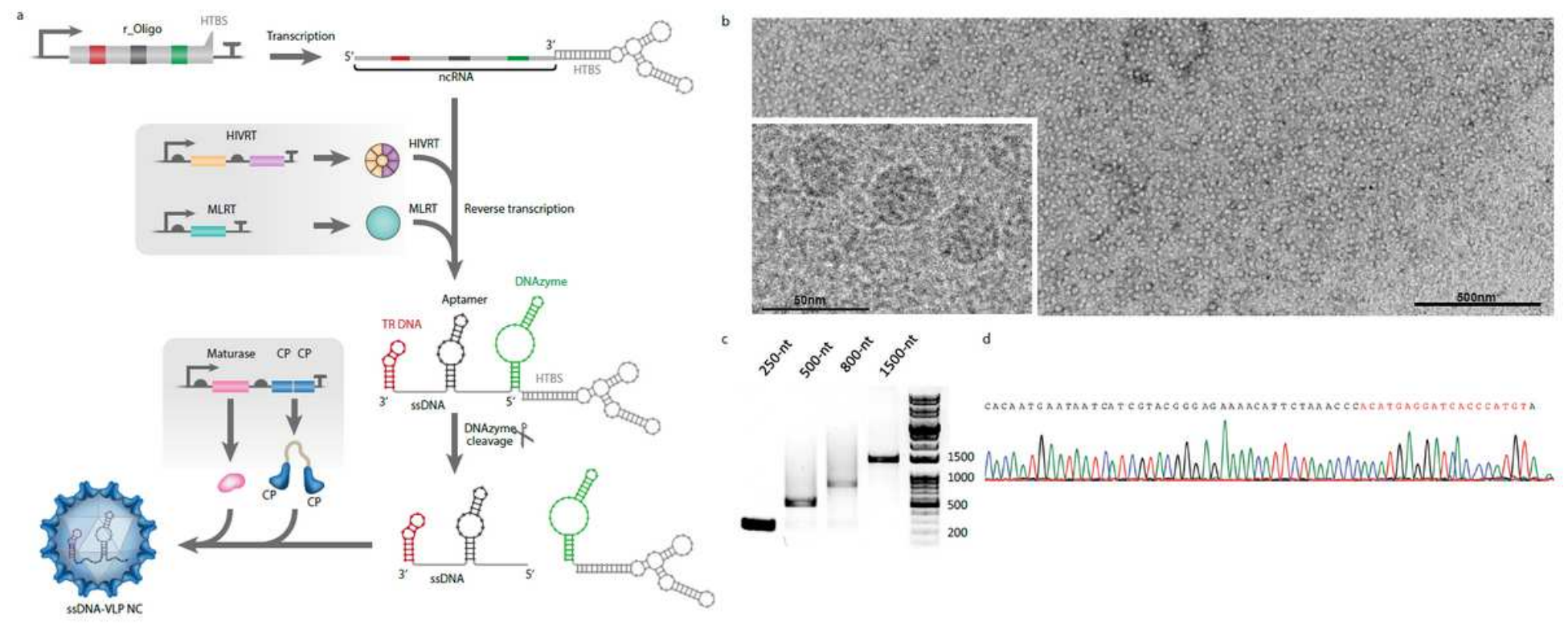

\section{Figure 4}

In vivo production of ssDNA-VLPs NCs. (a) Schematic representation of the genetically encoded in vivo production system's mechanism. The r_oligo gene contains (i) the desired ssDNA sequence, such as an aptamer (black), (ii) the TR-DNA activator sequence (red), (iii) the HIV reverse transcriptase binding site (HTBS), which serves as a terminator (grey), and (iv) self-cleaved DNAzyme which removes the HTBS following the conversion from RNA to DNA. (b) TEM images of the in vivo produced ssDNA-VLPs NCs. The inset represents the Cryo-TEM image of the same sample. (c) Gel electrophoresis of SsDNA sequences of different lengths, which were purified directly from the ssDNA-VLPs NCs. (d) Representative sequencing analysis of the in vivo produced ssDNA. The red-marked sequence represents the TR-DNA sequence. 


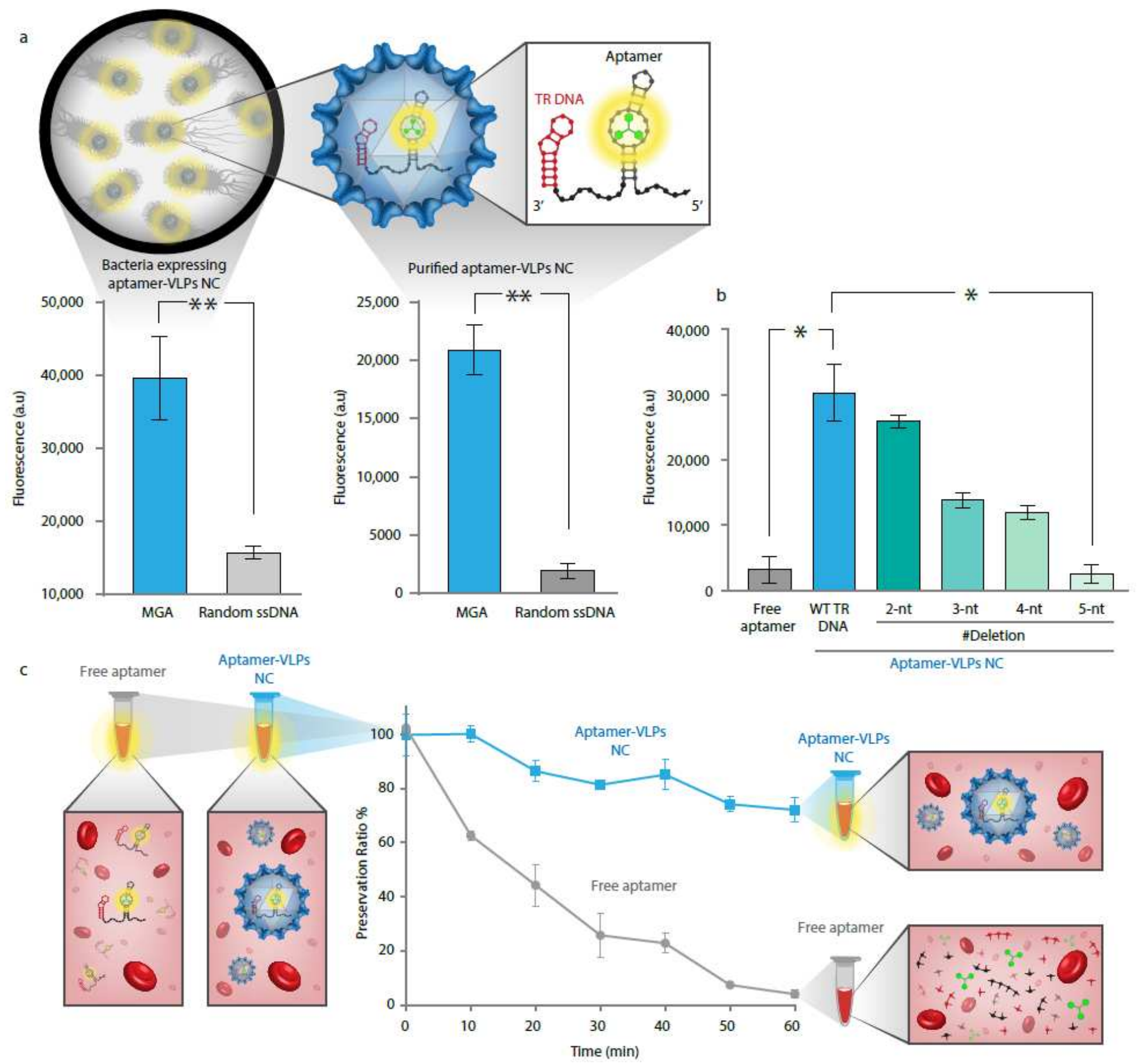

Figure 5

Application of the ssDNA-VLPs NCs under real-world conditions. (a) The fluorescence signal of functional aptamer-VLPs NCs within bacteria (left), and post purification (right). (b) Fluorescence signals of the TRDNA variants showing the impact of the TR-DNA activator sequence on SSDNA-VLPs NCs' assembly. ${ }^{*} P<0.01,{ }^{*} P<0.005$, (2-tailed Student's t-test). Error bars represent full experimental reproduction (3 repeats) of the process. (c) Comparison between the fluorescence signals at different time points of encapsulated MGA inside ssDNA-VLPs NCs and free MGA in 100\% human blood serum.

\section{Supplementary Files}


This is a list of supplementary files associated with this preprint. Click to download.

- SI.pdf

- Methods.pdf 